\section{La diagnosi precoce di malattia}

Giornale di Tecniche Nefrologiche e Dialitiche 2019, Vol. 3I (I) 58-60

(C) The Author(s) 2019

Article reuse guidelines:

sagepub.com/journals-permissions

DOI: | $0.1 \mid 77 / 039493621983749$

journals.sagepub.com/home/gtn

\author{
Federica Rossi e Federico Pieruzzi
}

\begin{abstract}
Early diagnosis in Fabry's disease

Aderson-Fabry disease is an X-linked, lysosomal, storage disorder characterized by the decreased activity of alphaGalactosidase A, which results in accumulation of globotriaosylceramide (Gb-3) in cells and tissues throughout the body, leading to a wide spectrum of clinical manifestations.

Patients are often misdiagnosed or diagnosed late in their life. This is due to the phenotypic heterogeneity, the poor awareness of this rare disease, and many pitfalls when making a differential diagnosis, in adulthood, as well as in the early stages.

Delayed diagnosis has significant clinical implications, because the progression of the disease over time can lead to irreversible end-stage renal disease and life-threatening cardiovascular or cerebrovascular complications.

Early diagnosis remains essential in order to start an early treatment and reduce the progression of the disease, thus maximizing the chance to improve patient outcomes.

Newborn screening, high-risk patients' identification, and increasing pediatricians' and clinicians' knowledge on this condition, are good strategies to avoid late referrals of Anderson-Fabry patients to reference centers.
\end{abstract}

\title{
Keywords
}

Fabry disease, X-linked, Screening, Treatment, Organ damage progression

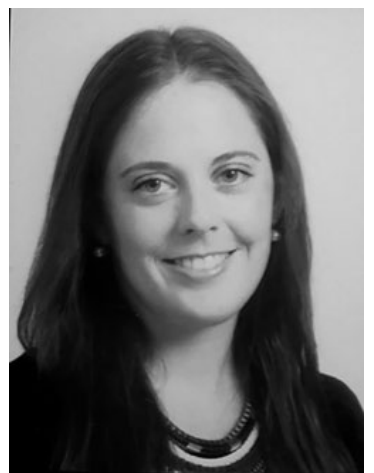

\section{Introduzione}

La Malattia di Anderson-Fabry è una patologia genetica X-linked dovuta alla mutazione del gene GLA (Xq21.3-q11), che comporta un deficit parziale o totale dell'enzima alfagalattosidasi A $(\alpha$ Gal-A $)$. A tale deficit consegue l'accumulo lisosomiale di alcuni sfingolipidi, prevalentemente globotriaosilceramide (Gb3), a

livello di differenti organi e tessuti quali cuore, rene e sistema nervoso centrale, che determinano un progressivo e irreversibile danno funzionale. ${ }^{1}$

La prevalenza mondiale della malattia varia in un range che va da un caso ogni 40,000 fino ad un caso ogni 60,000 abitanti. ${ }^{2}$ Tuttavia, vista la difficoltà diagnostica correlata all'aspecificità della presentazione clinica iniziale, possiamo ritenere questi dati nettamente sottostimati. Se valutiamo infatti due screening neonatali effettuati rispettivamente in Italia e in Taiwan, notiamo come la prevalenza risulti nettamente maggiore, con un paziente affetto ogni 3,100 nel primo caso $^{3}$ e uno ogni 1,500 nel secondo caso. ${ }^{4}$

\section{Perché una diagnosi precoce è difficile?}

Ad oggi sono state descritte oltre 800 mutazioni del gene GLA $^{5}$ che influenzano in modo differente l'attività dell'enzima $\alpha$ Gal-A dando luogo ad un'espressione fenotipica eterogenea che rende difficoltoso l'inquadramento diagnostico dei pazienti affetti.

S.C. Nefrologia ASST - Monza, Clinica Nefrologica, Dipartimento di Medicina e Chirurgia, Università degli Studi di Milano-Bicocca, Italy

\section{Corrispondenza:}

Dott. Federico Pieruzzi, Direttore f.f. S.C. Nefrologia ASST Monza, Clinica Nefrologica, Dipartimento di Medicina e Chirurgia, Università degli Studi di Milano-Bicocca, Ospedale San Gerardo, Via Pergolesi 33, 20900 Monza (MB), Italy.

E-mail: federico.pieruzzi@unimib.it 
I pazienti maschi omozigoti possono essere portatori di mutazioni nonsenso con totale assenza dell'enzima $\alpha$ GalA. Tali pazienti presentano un quadro clinico più grave, ad esordio precoce e coinvolgimento multisistemico. Generalmente questi soggetti, già in età pediatrica, giungono all'attenzione del medico per crisi acute di dolore urente localizzato alle estremità, non responsivo alla somministrazione di analgesici (acroparestesie), episodi di febbre di origine non definita, intolleranza al caldo, al freddo e all'esercizio fisico, ridotta sudorazione e alterazioni gastrointestinali con dolore addominale e turbe dell'alvo; tutti questi sintomi sono poco specifici e generalmente vengono ricondotti a patologie più comuni quali l'artrite reumatoide, distrofia muscolare, infezioni virali o, ancora, disturbi coinvolgenti la sfera psicologica o psicosomatica.

Esistono poi soggetti maschi che presentano mutazioni differenti (splicing, delezioni, inserzioni e mutazioni missenso in cui un aminoacido è sostituito da un altro) che, seppur in quantità ridotta, determinano un'attività' enzimatica residua e un fenotipo clinico più sfumato, a presentazione tardiva e che può interessare un solo organo (es. variante cardiaca). In questi casi è ancora più difficile giungere ad una definizione diagnostica.

Le pazienti femmine eterozigoti presentano un fenotipo clinico variegato in relazione al differente grado di inattivazione del cromosoma $\mathrm{X}$ nei diversi tessuti (lyonizzazione); in queste pazienti il danno d'organo dipende dal mosaicismo delle cellule nate sane e malate. ${ }^{6}$

Spesso, ciò che aiuta una diagnosi precoce, soprattutto nei pazienti di sesso maschile, è il riscontro di angiocheratomi cutanei diffusi a livello di pelvi, glutei e parte inferiore dell'addome, mentre nel sesso femminile gli angiocheratomi sono più rari e, allorquando presenti, meno estesi e di piccole dimensioni. La diagnosi può essere quindi guidata, in età più tardiva, intorno alla terza o quarta decade di vita dalla comparsa di proteinuria e insufficienza renale progressiva, possibile comparsa di stroke o riscontro di cardiomiopatia da accumulo associata ad alterazioni del ritmo cardiaco. ${ }^{7}$

In generale, si stima che dall'età di insorgenza della malattia fino alla sua individuazione passino circa 15 anni; ${ }^{8}$ anni in cui il paziente viene valutato da numerosi specialisti e sottoposto inutilmente a svariati esami di approfondimento prima di giungere ad una formulazione diagnostica corretta.

\section{Come confermare un sospetto diagnostico?}

Un sospetto clinico si può porre sulla base dell'anamnesi, esame obiettivo e valutazione della sintomatologia clinica; molte volte può essere d'aiuto la storia familiare del paziente.
La diagnosi definitiva viene posta, nel maschio emizigote, dal riscontro di un marcato deficit dell'enzima $\alpha$ Gal-A a cui viene fatto seguire un test genetico per l'individuazione della mutazione patogenetica.

Nella femmina eterozigote, invece, è sempre indicata l'esecuzione del test genetico da affiancare al dosaggio enzimatico, il quale può risultare, in una femmina affetta, sia nella norma (inattivazione bilanciata tra le due copie del gene) che deficitario (inattivazione sbilanciata a favore della copia mutata del gene). ${ }^{9}$

Una volta verificato che la mutazione sia patogenetica potremmo estendere lo screening a tutti i componenti suscettibili della famiglia, anticipando così le manifestazioni fenotipiche, garantendo una diagnosi precoce e il concomitante inizio della terapia mirata. Nelle varianti di significato incerto o nei polimorfismi, sarà comunque necessario verificare l'effettiva assenza di accumulo di Gb3 nei tessuti. Nel caso non fosse possibile eseguire l'indagine bioptica, sarà necessario eseguire il dosaggio del LysoGb3 e seguire i pazienti nel tempo sorvegliando l'eventuale comparsa di danno d'organo.

\section{Perché una diagnosi precoce è importante?}

Un ritardo nella formulazione di un sospetto clinico corretto determina un conseguente ritardo nell'intraprendere una terapia specifica mirata a stabilizzare e prevenire la progressione della malattia.

Quando instaurato, il danno d'organo clinicamente diagnosticabile risulta irreversibile. I dati per ora a nostra disposizione indicano come in fase avanzata di malattia, la terapia specifica non mostri capacità di modificare il coinvolgimento renale, cardiaco e del sistema nervoso. Al contrario, una maggiore efficacia si riscontra quando la terapia viene iniziata precocemente, in particolare nel rallentamento del quadro clinico, nella remissione dei sintomi, e nella prevenzione degli eventi maggiori. Pertanto, più precoce è la diagnosi, più precoce ed efficace può risultare la strategia terapeutica intrapresa.

\section{Necessità di uno screening neonatale}

Alla luce della variegata espressione fenotipica di questa malattia con quadri clinici molto sfumati e ad insorgenza tardiva che possono sfuggire alla diagnosi e alla necessità di iniziare precocemente la terapia, sarebbe auspicabile inserire anche la Malattia di Fabry nei protocolli di screening neonatale. In questa direzione si stanno muovendo numerose realtà internazionali e anche nazionali con il supporto delle associazioni dei pazienti. In Toscana, lo screening neonatale è già in atto ed i primi dati indicano che numerose famiglie diagnosticate possono finalmente essere inserite precocemente in un programma di follow-up clinico specifico. 


\section{Conclusioni}

Concludendo, è fondamentale sottolineare l'importanza di sensibilizzare i clinici ad una maggiore attenzione nell'osservazione clinica, soprattutto nell'età pediatrica, per porre la malattia di Fabry in diagnosi differenziale con le patologie più note. Un approccio importante ai fini della diagnosi precoce è insito nei programmi di screening neonatali. Tuttavia, l'identificazione del difetto genetico deve essere inevitabilmente associata ad una corretta diagnosi di patogenicità e di inquadramento fenotipico presso i centri di riferimento presenti sul territorio nazionale.

\section{Dichiarazione di assenza di conflitto di interessi}

Gli Autori dichiarano di non avere conflitti di interessi.

\section{Finanziamenti}

Gli Autori dichiarano di non aver ricevuto finanziamenti specifici da qualsiasi ente nei settori pubblico, privato o senza fini di lucro.

\section{Bibliografia}

1. Hoffmann B. Fabry disease: recent advances in pathology, diagnosis, treatment and monitoring. Orphanet J Rare Dis. 2009; 4:21.
2. Mehta A, Beck M, Eyskens F, et al. Fabry disease: a review of current management strategies. QJM 2010; 103(9):641-59.

3. Spada M, Pagliarini S, Yasuda M, et al. High incidence of later-onset Fabry disease revealed by newborn screening. Am J Hum Genet 2006; 79(1):31-40.

4. Hwu WL, Chien YH, Lee NC, et al. Newborn screening for Fabry disease in Taiwan reveals a high incidence of the lateronset GLA mutation c.936+919G $>$ A (IVS4+919G $>$ A). Hum Mutat 2009; 30(10):1397-405.

5. Human Gene Mutation Database website, https://portal. biobase-international.com (ultimo accesso 5 novembre 2018).

6. Gupta S, Ries M, Kotsopoulos S, et al. The relationship of vascular glycolipid storage to clinical manifestations of Fabry disease: a cross-sectional study of a large cohort of clinically affected heterozygous women. Medicine (Baltimore) 2005; 84(5):261-8.

7. Garcia de Lorenzo A; en nombre del grupo de endermedad de FABRY-GETER. Consensus for the study and treatment of Fabry disease. GETER Foundation. Med Clin (Barc) 2011;137(4):178-83.

8. Germain DP. Fabry Disease. Orphanet J Rare Dis 2010; 5:30.

9. Hagège AA, Caudron E, Damy T, et al. Screening patients with hypertrophic cardiomyopathy for Fabry disease using a filter-paper test: the FOCUS study. Heart. 2011; 97(2):131-6. 